\title{
La participation, une légitimité en question
}

\author{
Cécile Barnaud \\ Géographe, INRA, SAD UMR DYNAFOR, 31326 Castanet-Tolosan, France
}

Les démarches participatives ne sont pas un sujet nouveau pour Natures Sciences Sociétés (NSS, 18, 4, 395-403 ; NSS, 20, 1, 52-65 ; NSS, 20, 3, 286-296 ) et il est intéressant de noter que ce sujet devient très courant dans le monde scientifique depuis quelques années à propos de dispositifs qui associent des amateurs ou de simples citoyens à des protocoles de recherche. Dans cet article, l'auteure s'interroge sur les fondements de la légitimité sociale de ces recherches à partir de deux expériences qu'elle a conduites en Thaïlande. Dans l'esprit de la démarche ComMod (NSS, 13, 2, 165-168), elle a recours à la modélisation comme outil de facilitation de l'expression et de la formalisation des connaissances ainsi que des intérêts des différentes parties prenantes. Son travail la conduit à examiner la question des asymétries de pouvoir entre les participants dans ce type de démarches.

La Rédaction

\section{Mots-clés :}

environnement ; gouvernance ; participation ; pouvoir ; réflexivité

\section{Keywords:}

environment; governance; participation; power; reflexivity
Résumé - La légitimité des démarches participatives est une question récurrente mais rarement abordée de front, qui présente des dimensions à la fois sociales, culturelles et scientifiques. Cet article interroge en particulier la légitimité sociale de ces démarches dont la neutralité est illusoire, et dont les animateurs sont confrontés à un dilemme lorsque se pose la question de leur positionnement vis-à-vis des asymétries de pouvoir. En effet, celui qui adopte une posture neutre risque de voir les acteurs les plus influents dominer le processus, mais d'un autre côté, celui qui intervient en faveur des acteurs les moins influents n'en a pas forcément la légitimité. Adoptant une démarche de recherche impliquée, ce travail propose et met à l'épreuve sur deux terrains une posture d'accompagnement critique qui tente de surmonter ce dilemme, en concevant la légitimité comme le fruit d'une coconstruction, en explicitant ses partis-pris pour qu'ils puissent être remis en cause. L'analyse réflexive de ces deux expériences nous permet de débattre des limites de cette posture et d'approfondir la question de la légitimité sociale de la participation, tout en interrogeant ses dimensions culturelles et scientifiques.

\begin{abstract}
Participation: legitimacy in question. Participatory approaches are widely used nowadays, but some questions regarding their legitimacy remain poorly addressed. The legitimacy of participatory processes involves several dimensions: legitimacy of the persons driving the processes, legitimacy of the tools they use, legitimacy of the knowledge they produce, etc. This paper aims to question the social legitimacy of these participatory processes, considering that their neutrality is illusionary, especially regarding the way to deal with power asymmetries among stakeholders. On the one hand, those who adopt a neutral position run the risk of being manipulated by the most powerful local stakeholders while on the other hand those who voluntarily empower the powerless stakeholders rarely have the legitimacy to do so. In the face of this dilemma, our paper examines the testing of a "critical companion posture" which recognizes the need to take into account the local stakeholders' power games to avoid the risk of increasing initial inequalities, and which considers legitimacy the result of a co-construction process: the designer should attempt to make explicit all his/her underlying assumptions so that local stakeholders can choose to accept them as legitimate or to reject them. The paper draws on the experimentation and reflexive analysis of two companion modelling processes conducted with this critical posture in the highlands of Northern Thailand. The discussion about the limits of this posture allows us to delve further into the question of the social legitimacy of participation. These two experiments also enable us to address the cultural and scientific dimensions of this legitimacy.
\end{abstract}

Auteur correspondant : cecile.barnaud@toulouse.inra.fr 


\section{Introduction}

Les démarches dites participatives connaissent depuis deux décennies un essor considérable, notamment dans les domaines du développement rural et de la gestion des ressources renouvelables où il s'agit de permettre aux " acteurs locaux » (citoyens, usagers des ressources) de participer à l'élaboration des projets et politiques les concernant (Chambers et al., 1989 ; BorriniFeyerabend et al., 2004 ; Johnson et al., 2004) ${ }^{1}$. Ces nouvelles formes de dialogue entre des acteurs hétérogènes se concrétisent par la mise en place d'arènes multiacteurs plus ou moins pérennes, généralement pilotées par un ou plusieurs individus (appelés animateurs, facilitateurs ou médiateurs). Dans le cadre de démarches de rechercheintervention destinées à produire des connaissances avec et pour les acteurs locaux, les chercheurs sont de plus en plus souvent amenés à prendre part à ces arènes, dont ils occupent même parfois la position d'animateur (Casabianca et Albaladejo, 1997; Chanal et al., 1997; Soulard et al., 2007 ; Petit et al., 2008 ; Mormont, 2009).

Si elles se multiplient, ces démarches font également l'objet d'un nombre croissant de critiques (LavigneDelville et al., 2000 ; Cooke et Kothari, 2001 ; Barbier, 2005), notamment quant à leur légitimité : légitimité des partis-pris qui sous-tendent ces démarches, de ceux qui les pilotent, des outils qu'ils mobilisent, des individus qui y participent, des actions qui en découlent, ou encore des connaissances qu'elles produisent. Cependant, la question de la légitimité n'est que rarement abordée de front (Casabianca et Albaladejo, 1997 ; Daré et al., 2008 ; D'Aquino, 2009; Barnaud et al., 2010a). C'est en effet une question délicate $\mathrm{du}$ fait $\mathrm{du}$ caractère subjectif $\mathrm{du}$ concept de légitimité : ce qui est légitime aux yeux des uns ne l'est pas forcément aux yeux des autres. Cet article propose d'appréhender concrètement cette question en considérant la légitimité comme le « fruit interactionniste et évolutif de compromis entre des principes et des valeurs qui aboutissent progressivement à une convention à laquelle les acteurs font appel pour justifier leurs choix » (Boltanski et Thévenot, 1991).

La légitimité des démarches participatives peut être interrogée dans plusieurs dimensions : légitimité sociale d'un processus contribuant à modifier les rapports sociaux dans une société, légitimité culturelle de processus conduits hors du berceau culturel des idéologies qui sous-tendent le principe de participation, légitimité scientifique des connaissances produites dans ces processus. Le travail présenté ici est centré sur la question de la légitimité sociale de la participation - question qui se

Ces travaux ont été réalisés dans le cadre d'une thèse de doctorat en Géographie humaine, économique et régionale à l'Université Paris X, en accueil à l'UPR Green du Cirad, et basée à l'Université de Chulalongkorn à Bangkok (Thaïlande). pose que le processus soit piloté par un agent de développement ou par un chercheur -, mais dans la mesure où le travail repose sur deux processus participatifs pilotés par une chercheuse occidentale dans un pays d'Asie $\mathrm{du}$ Sud-Est, les dimensions scientifiques et culturelles y sont également abordées.

La dimension sociale de la légitimité renvoie à la critique fréquente du manque de prise en compte de la complexité des contextes sociopolitiques dans lesquels les dispositifs participatifs sont mis en œuvre (Nelson et Wright, 1995 ; Lavigne-Delville et al., 2000 ;Eversol, 2003 ; Mermet et al., 2005 ;Munoz et al., 2007). Ces contextes sont en effet souvent caractérisés par des conflits d'intérêts entre des acteurs qui n'ont ni la même capacité, ni le même intérêt à participer au processus, à se l'approprier ou à en influencer le cours. Tout processus participatif peut donc contribuer, volontairement ou non, à modifier les rapports de force entre acteurs, et pose donc la question de la légitimité sociale du processus et de celui qui le pilote. Cependant, face à cette question, ces pilotes sont confrontés à un dilemme. D'un côté, s'ils mettent en avant leur neutralité face aux asymétries de pouvoir, ne souhaitant pas prendre parti pour l'un ou l'autre des acteurs en présence, on leur reproche d'être naïvement manipulés par les acteurs les plus influents et de participer en fait à un renforcement des inégalités existantes (Cooke et Kothari, 2001 ; Edmunds et Wollenberg, 2001). Leur neutralité serait donc illusoire. Mais, d'un autre côté, s'ils assument leur non-neutralité en intervenant en faveur d'un groupe d'acteurs donné, on peut interroger leur légitimité à modifier ainsi les rapports de force au sein d'un système social (Innes, 2004 ; D'Aquino, 2009).

Le travail présenté dans cet article propose et teste une posture particulière qui tente de surmonter ce dilemme. Cette posture, qualifiée d'accompagnement critique, est une posture explicitement non neutre qui prend volontairement en compte les asymétries de pouvoir de façon à éviter que le processus n'accroisse les inégalités initiales. Elle repose sur un effort d'explicitation des partispris qui lui sont associés, de manière qu'ils puissent être remis en cause ou au contraire légitimés par les participants. La légitimité du processus est ainsi coconstruite au fil du temps. L'objectif de cet article n'est pas de défendre l'idée que cette posture est la «bonne » posture (nous ne pensons pas qu'une telle posture existe), mais d'analyser la portée et les limites d'une posture particulière pour contribuer à faire avancer les réflexions sur la légitimité sociale des démarches participatives.

\section{Asymétries de pouvoir et participation : cadrage théorique}

Si la façon dont les pilotes de démarches participatives se positionnent vis-à-vis des asymétries de pouvoir n'est 
jamais neutre, ce positionnement n'est que rarement explicité. Dans cette première partie, nous proposons un cadrage théorique qui permet de présenter les débats théoriques sous-jacents à ces questions et d'expliciter les fondements de la posture proposée dans cet article.

\section{Postures dialogiques vs postures critiques}

La littérature relative aux démarches participatives distingue fréquemment les tenants des postures dialogiques de ceux des postures critiques (Faysse, 2006), inspirées respectivement des approches des soft systems (Checkland, 1981) et des critical systems (Ulrich, 1983; Jackson, 2000). Pour les premiers, c'est l'existence de différentes interprétations possibles d'un problème par différents acteurs qui constitue le principal obstacle à l'émergence d'une collaboration fructueuse entre ces acteurs. La solution réside donc dans le dialogue pour une compréhension mutuelle accrue (Pretty, 1998). Pour les seconds, les approches dialogiques sont insuffisantes dans les situations conflictuelles et coercitives, car elles ne permettent pas que soit prise en compte la diversité des intérêts en présence, notamment ceux des acteurs les moins influents (Edmunds et Wollenberg, 2001). Celui qui pilote un dispositif participatif devrait donc selon eux agir stratégiquement sur l'espace d'échanges pour éviter qu'il ne soit dominé par les acteurs les plus influents. Alors que les auteurs dialogiques considèrent le consensus comme une condition nécessaire à l'action collective, les auteurs critiques considèrent que le consensus est souvent illusoire, ne reflétant que les opinions des plus influents, et préfèrent souligner la pluralité des intérêts en présence (Wollenberg et al., 2001).

Ces débats reposent en partie sur les travaux du philosophe Habermas (1987). Selon cet auteur, le résultat d'un processus de concertation devrait être basé, dans l'idéal, sur des arguments et non sur des rapports de force. Il définit un ensemble de conditions à réunir pour que s'établisse une telle "situation idéale de parole", dans laquelle chaque sujet est doté de la même capacité à participer aux discussions (mêmes droits, mêmes informations, mêmes opportunités). Toutes les formes d'asymétrie et de coercition dans le dialogue sont considérées comme des «distorsions communicationnelles». Les auteurs dialogiques sont souvent, explicitement ou non, associés au cadre de pensée d'Habermas. À l'inverse, la plupart des auteurs critiques se situent en opposition à ses travaux, dénonçant le caractère utopique de la situation idéale de parole, et le risque associé de minimiser les asymétries entre les acteurs (Innes, 2004). Cependant, certains auteurs critiques considèrent au contraire que les travaux d'Habermas incitent à mettre en évidence les distorsions communicationnelles, et donc les asymétries de pouvoir (Ulrich, 1983 ; Leeuwis, 2000). Cette lecture permet de concevoir les approches dialogiques et critiques comme étant complémentaires et non pas antagonistes.
La prise en compte stratégique des asymétries de pouvoir (prônée par les auteurs critiques) peut permettre en effet de réduire les distorsions communicationnelles au sein d'une arène de concertation, de façon à favoriser les conditions d'émergence d'un dialogue et d'une plus grande compréhension mutuelle (prônés par les dialogiques). C'est sur cette complémentarité que repose la « posture d'accompagnement critique » discutée dans cet article.

\section{La posture de la modélisation d'accompagnement}

Cette posture s'inscrit également dans le cadre de la démarche de modélisation d'accompagnement (démarche ComMod, pour Companion Modelling), fondée sur l'usage de modèles pour faciliter des processus d'apprentissage collectif entre acteurs (Bousquet et al., 1996). L'une de ses originalités réside dans l'existence d'une charte qui explicite une posture participative particulière (ComMod, 2005). Bien que plusieurs chercheurs ComMod aient abordé la question de la prise en compte du contexte social (Daré et al., 2007 ; Becu et al., 2008; D'Aquino, 2009), la charte ne se positionne pas explicitement sur la façon de gérer les asymétries de pouvoir. Une étude récente a d'ailleurs montré que les chercheurs mettant en œuvre cette démarche adoptent à ce niveau des postures diverses, tant dialogiques que critiques (Barnaud et al., 2010a). En revanche, l'un des éléments fondamentaux de la posture explicitée dans la charte est la volonté de «n'avoir aucune hypothèse implicite dans l'expérimentation » (ComMod, 2005). Ceci implique de la part de celui qui conduit un processus ComMod un effort systématique d'explicitation de ses hypothèses auprès des participants. Les hypothèses dont il est question dans la charte concernent au premier plan les hypothèses sous-jacentes au modèle coconstruit avec les acteurs. Cependant, elles peuvent être étendues à l'ensemble des hypothèses sous-jacentes au processus mis en œuvre (pourquoi choisir tel outil, inviter tel participant, mettre l'accent sur telle question), et notamment celles qui concernent le positionnement vis-à-vis des asymétries de pouvoir. C'est ce qui est proposé dans la posture d'accompagnement critique, qui nous amènera donc à poser la question : est-il possible d'être à la fois stratégique et transparent?

\section{Une posture d'accompagnement critique}

Il s'agit d'une posture critique qui considère nécessaire de prendre en compte les asymétries de pouvoir entre les acteurs, pour éviter que le processus participatif conduit n'accroisse les inégalités initiales. Adopter une posture neutre est considéré comme problématique, non seulement parce qu'en fermant les yeux sur les inégalités, la démarche risque de les renforcer, mais aussi parce qu'en se considérant comme neutre, le pilote d'une 
démarche participative n'explicite pas ses partis-pris et risque de les imposer inconsciemment, sans qu'ils puissent être remis en cause. En se référant aux principes de l'approche ComMod, la posture d'accompagnement critique suggère que le pilote $d^{\prime}$ un processus participatif explicite les partis-pris sous-jacents à sa démarche, de sorte que les acteurs puissent les remettre en cause ou au contraire les accepter comme étant légitimes. L'adhésion des acteurs à la démarche proposée est considérée comme sa principale source de légitimité. En accord avec la définition de Boltanski et Thévenot, la légitimité de la démarche est ici le fruit d'une coconstruction itérative et adaptative entre les acteurs et le pilote de la démarche, qui explicite ses objectifs et les ajuste si nécessaire au fil de discussions avec les acteurs.

La posture d'accompagnement critique se donne ainsi pour objectif explicite de faciliter l'émergence d'une forme d'échanges qualifiée de concertation équitable. L'équité est ici entendue au sens de Rawls (1997), c'est-à-dire d'égalité de chances. Mais alors que cet auteur se réfère à une équité sociale au sein d'une société, il s'agit ici d'une équité procédurale au sein de l'arène de concertation. Un processus de concertation équitable est donc ici défini comme un processus dans lequel tous les protagonistes ont une chance de faire entendre et valoir leurs intérêts, l'égalité de chances étant un idéal visé dont on peut, au mieux, tenter de se rapprocher. À l'instar de la situation idéale de parole, il s'agit d'un idéal théorique dont le principal intérêt est de souligner l'existence d'obstacles pour l'atteindre, afin d'essayer de les surmonter.

\section{Méthode : une démarche de recherche impliquée}

La démarche de recherche choisie fut celle d'une recherche impliquée. Nous avons mis en œuvre deux processus participatifs dans le nord de la Thaillande en adoptant une posture d'accompagnement critique, avant de procéder à l'analyse réflexive de ces deux expériences.

\section{Contexte de mise en œuvre}

Pour débattre de la légitimité d'un processus participatif, il est important d'expliciter les conditions d'initiation de ce processus (Mathevet et al., 2010). A-t-il été mis en œuvre dans un contexte institutionnel porteur? Qui l'a initié ? Qui l'a soutenu ? Qui en a formulé les objectifs ? Cette section répond brièvement à ces questions.

Lorsque les expériences ont été mises en œuvre, entre 2005 et 2007, la Thailande connaissait depuis une décennie une tendance politique décentralisatrice prônant une plus grande participation des acteurs locaux à la gestion des ressources naturelles - eau, forêt, foncier - (Arghiros,
2001 ; Sato, 2003). Cela s'était notamment traduit en 1994 par une réforme des administrations décentralisées à l'échelle du sous-district, avec la constitution de conseils comprenant deux élus par village, et le renforcement des moyens alloués à des projets locaux. Dans le contexte du nord de la Thaillande, ces politiques ont offert aux communautés montagnardes une opportunité sans précédent d'avoir davantage voix au chapitre dans la gestion des ressources locales. Cette région est en effet peuplée de minorités ethniques qui ont longtemps été accusées de dégrader les hauts bassins versants des grands cours d'eau du pays, et dont l'accès aux ressources a été restreint, notamment à travers la mise en place de parcs nationaux. Le contexte institutionnel national et régional était donc porteur d'une volonté de changement et de promotion d'approches participatives, ce qui explique que des appuis ont été trouvés localement aux expériences menées dans le cadre de ce travail. Le choix d'axer nos travaux sur la question des asymétries de pouvoir se justifie par le constat fait par de nombreux auteurs d'un risque d'accaparement des projets participatifs par les élites locales, phénomène amplifié par la prégnance locale d'un "mythe de la communauté homogène» (Guijt et Shah, 1998), que certains qualifient d'« ethnoromantisme » (Neef, 2005).

La première expérience a été menée dans la continuité d'une démarche ComMod, initiée en 2002 par une équipe de chercheurs franco-thaillandaise, dont l'objectif était de valider auprès des agriculteurs leurs connaissances au sujet de l'érosion des sols (Trébuil et al., 2002). La démarche était soutenue par deux membres d'un bureau local d'une agence gouvernementale de développement, mais aucune demande sociale n'avait été formulée. Lorsque les participants se sont familiarisés avec la démarche et les outils proposés, ils ont demandé aux chercheurs de venir traiter de questions plus proches de leurs préoccupations. C'est ainsi qu'ils ont demandé successivement $\mathrm{d}^{\prime}$ aborder les questions de règle $\mathrm{d}$ 'accès au crédit (pour l'investissement dans des cultures pérennes non érosives) [Barnaud et al., 2007], puis de gestion de l'eau d'irrigation (pour irriguer ces cultures pérennes en pleine expansion) [Barnaud et al., 2008b]. C'est de ce troisième cycle dont il est question ici.

La question de l'eau a émergé dans le cadre d'une tension croissante liée aux règles d'accès à l'eau au sein de la communauté. La règle en vigueur dans ce village était celle du «premier arrivé, premier servi » : une fois qu'un agriculteur avait installé sa prise d'eau dans un ruisseau pour capter l'eau par gravité, aucun autre ne pouvait venir placer la sienne en amont. De ce fait, seule une minorité d'agriculteurs appartenant aux clans les plus aisés et les plus influents du village avait accès à l'eau. Dans un contexte d'augmentation du nombre de prétendants à l'irrigation, un leader du village proposa la mise en place d'un processus ComMod au sujet de l'eau, en 
suggérant d'y associer l'administration décentralisée du sous-district, censée soutenir et financer des projets hydro-agricoles locaux. L'objectif du processus était donc de faciliter une concertation au sujet de l'eau entre les agriculteurs de la communauté, d'une part, et entre eux et l'administration du sous-district, d'autre part.

La seconde expérience fut conduite dans le contexte $d^{\prime}$ un conflit ouvert entre un parc national en cours d'établissement et deux communautés de Mien localisées en bordure du parc. Le parc national avait pour mission de protéger la forêt des hauts bassins versants du pays, alors que les communautés montagnardes étaient dépendantes de ces forêts, la collecte des produits forestiers non ligneux constituant une part importante de l'alimentation et du revenu des familles les plus pauvres (Barnaud et al., 2008a). Officiellement, toute activité humaine autre que le tourisme est interdite dans l'enceinte des parcs nationaux thaïlandais. Cependant, dans la pratique, des arrangements informels sont observés. Mais dans le cas présenté ici, la situation était si conflictuelle qu'aucun dialogue n'avait pu être établi entre les villageois et le directeur du parc, ni sur la question des futures frontières du parc ni sur celle des règles qui y seraient appliquées. Jusqu'à la mise en place du parc, c'était le département royal des forêts qui gérait les forêts de la zone. Après plusieurs décennies de gestion autoritaire des ressources et de conflits violents avec les villageois, ces forestiers avaient opté pour des modes de gestion plus participatifs et essayaient de convaincre le directeur du parc d'adopter la même attitude. Ce sont eux qui soutenaient la proposition de mettre en place un processus ComMod pour tenter d'établir un dialogue entre le parc et les villageois.

\section{Produire des connaissances par, sur et pour l'action}

Nous ne nous attarderons pas ici sur la justification générale des démarches de recherche-action et de recherche-intervention que le lecteur trouvera dans d'autres écrits (Casabianca et Albaladejo, 1997 ; Liu, 1997 ; Avenier et Schmitt, 2007 ; Petit et al., 2008 ; Stassart et al., 2008). En résumé, face à des problèmes complexes, caractérisés par des incertitudes et des enjeux sociaux tels que la définition même des problèmes fait l'objet de débats, ces démarches prônent la mise en place d'un dialogue entre chercheurs et citoyens (Funtowicz et Ravetz, 1994). Il s'agit de mener une recherche conduite avec et pour les acteurs afin de produire des connaissances dites « actionnables » (Argyris, 1995), utiles à l'action. Dans les démarches présentées ici, il s'agissait de produire des connaissances sur et par la gestion concertée et participative des ressources renouvelables. Deux types de connaissances ont été produites : (i) des connaissances générées dans l'action, issues d'une dynamique d'apprentissage collectif associant acteurs et chercheurs, sur les enjeux locaux de la gestion sociale des ressources, et (ii) des connaissances issues de l'analyse réflexive des deux processus participatifs, sur les jeux de pouvoir dans les démarches participatives : comment s'expriment les jeux de pouvoir dans les dispositifs participatifs ? Quelle est l'influence des choix méthodologiques (choix des supports, des participants, des méthodes d'animation, etc.) sur ces jeux de pouvoir? Qu'est-ce que cela implique quant à la légitimité des processus mis en œuvre?

Une telle démarche de recherche impliquée permet de comprendre les choses en les vivant de l'intérieur, mais elle nécessite une prise de recul pour l'analyse. Celle-ci a été facilitée dans notre cas par l'utilisation d'un journal de bord, dans lequel les faits étaient relatés et les choix explicités à chaud; le temps long (plusieurs mois) entre l'action et l'analyse; de nombreuses discussions avec $d$ 'autres chercheurs - impliqués et non impliqués dans l'expérience - ; et enfin l'usage d'un cadre d'analyse théorique, que nous présentons brièvement ci-dessous.

\section{Un cadre d'analyse ad hoc}

Ce cadre étant présenté en détail par ailleurs (Barnaud et al., 2010b), nous ne l'abordons ici que dans ses grandes lignes. Pour caractériser la situation initiale sont mobilisés des concepts issus de la théorie des systèmes agraires (Mazoyer et Roudart, 1997) et de l'analyse institutionnelle (Ostrom et al., 1994). Ils sont utilisés ici pour étudier, $d^{\prime}$ 'une part, les modes d'interaction entre les acteurs et, d'autre part, leurs différentes perceptions des situations, $l^{\prime}$ analyse de système agraire permettant $d$ 'aborder finement les perceptions de différents types d'agriculteurs, en fonction de leurs intérêts et de leurs pratiques. En outre, pour analyser la dynamique et les effets du processus ComMod, nous avons combiné une entrée par les relations de pouvoir (Giddens, 1984 ; Boulding, 1989), pour le suivi des interactions entre acteurs, avec une entrée par les théories de l'apprentissage et de la négociation (Leeuwis, 2000), pour le suivi de leurs perceptions. Alors que les tenants des postures dialogiques analysent classiquement les processus participatifs en termes d'apprentissage collectif (Röling et Wagemakers, 1998), la prise en compte explicite des jeux de pouvoir nous conduit à mobiliser aussi les théories de la négociation. Nous leur empruntons notamment la distinction entre dispositifs de négociation distributifs et intégratifs (Carnevale, 2006). Dans un processus de négociation distributif, les acteurs se confrontent sur la façon dont ils vont « se partager le gâteau » dans un jeu à somme nulle. Dans un processus intégratif, ils reformulent le problème de façon à «élargir le gâteau », ouvrant la voie à des jeux à somme positive. Un tel processus est plus exigeant en termes de changement cognitif, les acteurs devant faire l'effort de mieux comprendre les intérêts et les valeurs sous-jacents à leurs propres positions et à celles des autres. 


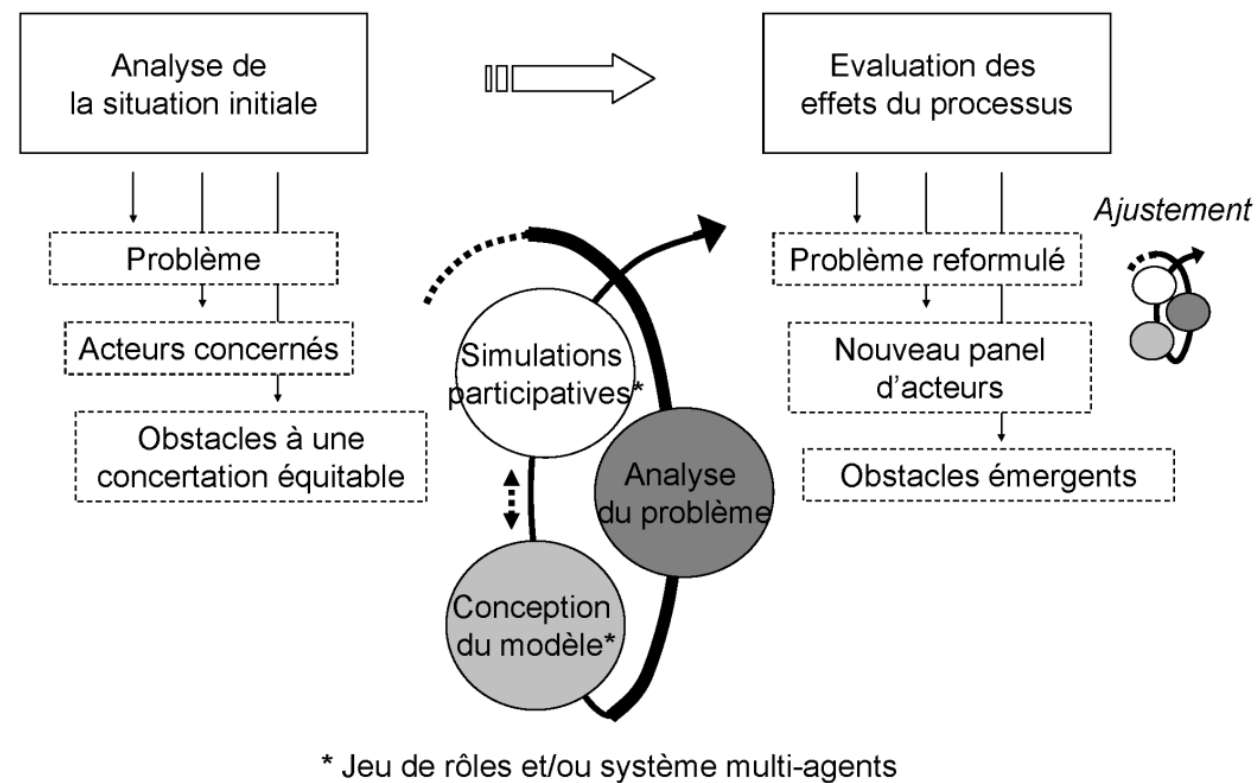

Fig. 1. Mise en œuvre des processus ComMod dans une posture d'accompagnement critique.

\section{Récit de deux processus participatifs}

\section{Méthode et outils mobilisés : deux processus ComMod}

L'une des particularités de la démarche de modélisation d'accompagnement est l'usage de modèles de simulation comme supports d'un apprentissage entre acteurs, chercheurs compris (Bousquet et al., 1996 ; ComMod, 2005). La démarche repose ainsi sur la coconstruction de modèles (modèles multiagents et/ou jeux de rôles) intégrant les différents points de vue en présence, qui sont ensuite utilisés pour l'exploration concertée de scénarios (Barreteau et al., 2001 ; Bousquet et al., 2002).

Les méthodes et les outils mobilisés dans nos deux expériences ayant été décrits par ailleurs (Barnaud et al., 2008a ; Barnaud et al., 2008b), nous ne les présentons ici que brièvement. Les deux processus ont débuté par une analyse initiale approfondie du contexte, à l'aide d'entretiens individuels semi-directifs auprès d'une trentaine d'acteurs clefs sur chaque terrain (agriculteurs, administrations, etc.). Puis, la mise en œuvre des processus ComMod fut cyclique et itérative, s'adaptant aux évolutions $\mathrm{du}$ contexte et des processus d'apprentissage suscités (Fig. 1), ponctuée d'ateliers collectifs combinant :

- des sessions de jeux de rôles dans lesquels les participants (entre 15 et 30) étaient mis en situation en jouant leur propre rôle ;

- des discussions en séances plénières et en sousgroupes sur les problèmes mis en évidence et les possibles solutions ;

- des simulations informatiques pour discuter des effets à long terme des solutions proposées ;
- des entretiens individuels permettant de mieux comprendre les comportements observés au cours des ateliers.

Un suivi-évaluation des effets des processus ComMod fut mené par l'équipe d'animation tout au long des deux expériences, à l'aide d'entretiens individuels semi-directifs et par une observation participante au sein des villages.

\section{Analyse des dynamiques de pouvoir et de négociation entre les acteurs}

L'analyse initiale nous a permis tout d'abord d'identifier un certain nombre d'obstacles à l'émergence de processus de concertation équitables (Barnaud et al., 2008a) : des obstacles horizontaux (au sein des villages) et verticaux (entre villages et administrations). Au niveau horizontal, sur les deux sites, les villageois qui étaient potentiellement les plus affectés par l'issue des concertations étaient également ceux qui avaient la plus faible capacité à défendre leurs intérêts. Il s'agissait dans les deux cas des acteurs socioéconomiques les plus vulnérables : les petits agriculteurs n'ayant pas accès à l'eau d'irrigation dans le premier site (Mae Salaep), et les villageois dépendants de la collecte des produits forestiers dans le second (Nan). En outre, ces acteurs politiquement faibles n'étaient pas forcément soutenus par leurs représentants villageois, surtout lorsque ces derniers avaient leurs propres intérêts à défendre. C'est ce que Ribot (2001) appelle un manque d'imputabilité vers le bas (downwards accountability) des représentants. Quant aux obstacles verticaux, ils provenaient dans les deux cas d'une longue tradition de gestion centralisée des ressources en Thaillande, avec d'un côté des villageois qui, 
habitués à un rôle passif auprès des administrations, n'avaient pas confiance en leur capacité à participer à la vie politique locale, et de l'autre des administrateurs qui, malgré leurs discours, restaient peu enclins aux principes de la participation et gardaient au final entre leurs mains tout pouvoir de décision.

Nous avons ensuite analysé la façon dont ces obstacles se sont exprimés dans les deux processus participatifs, et notamment les mécanismes par lesquels les acteurs les plus influents ont usé ou tenté d'user de leur pouvoir. Par exemple, dans les deux expériences, les chefs de village ont systématiquement suggéré de ne pas inviter les villageois les plus pauvres "parce qu'ils ne comprennent rien et qu'ils ne parlent jamais dans les réunions de toute façon ». Par ailleurs, dans les réunions plénières du processus participatif, il était fréquent d'observer que des participants n'osaient pas ouvertement remettre en cause une proposition faite par un leader villageois, et encore moins celle d'un agent d'une administration, laissant donc place à un consensus apparent.

L'analyse nous a permis de mettre en évidence les mécanismes par lesquels certains de ces obstacles ont pu être surmontés, au moins dans une certaine mesure. Les deux expériences ont en effet eu en commun de générer des processus de concertation favorisant une meilleure prise en compte des intérêts des acteurs les moins influents au sein des communautés. Ces expériences ont permis aux participants de prendre conscience de leur interdépendance vis-à-vis des problèmes abordés et de l'urgence d'en débattre collectivement. Elles ont favorisé une compréhension mutuelle accrue et une reformulation (reframing) des problèmes et des enjeux permettant l'exploration de voies de négociation intégratives. À Mae Salaep, les paysans sans accès à l'eau, soutenus par un leader religieux charismatique, ont ainsi proposé de construire des petits barrages sur les ruisseaux du bassin versant pour augmenter le nombre de plantations irrigables. L'habileté de cette proposition résidait dans son caractère intégratif qui a permis qu'elle soit acceptée par les acteurs des clans les plus influents : tout le monde a intérêt à ce que l'on augmente la quantité d'eau disponible. Pour les agriculteurs sans accès à l'eau, la construction de nouvelles infrastructures était surtout un prétexte pour redéfinir les règles de partage de l'eau (Barnaud et al., 2010b). À Nan, les chefs de village prirent conscience de leur intérêt à négocier, avec les agents du parc national, les droits de collecte des produits forestiers non ligneux dans l'enceinte du parc (problème spécifique aux villageois les plus pauvres). Alors qu'ils étaient bloqués dans un conflit sur la question des frontières, reformuler le problème en focalisant non plus sur les frontières mais sur les règles d'accès aux ressources leur permettait d'apaiser les relations avec le parc et augmentait leurs chances d'obtenir ultérieurement gain de cause sur d'autres sujets.
Si les obstacles horizontaux furent surmontés, au moins en partie, verticalement tous les blocages ne purent être levés. Dans les deux études de cas, alors que des accords furent trouvés au sein des communautés, les propositions des villageois ne furent pas réellement prises en compte par les acteurs aux niveaux supérieurs. À Mae Salaep, la présidente de l'administration du sousdistrict refusa de financer le projet que les villageois avaient rédigé suite aux ateliers; tandis qu'à Nan, le directeur du parc ne se rendit pas à l'atelier final où il aurait pu entamer un dialogue avec les villageois.

\section{Identification des choix méthodologiques non neutres et de leurs effets}

L'analyse réflexive nous a conduite par la suite à relever les principaux choix méthodologiques destinés à favoriser une concertation équitable, et dont on fait l'hypothèse qu'ils y ont contribué, au moins partiellement. Nous ne parlons ici que d'hypothèse car il est très difficile, dans l'évaluation des effets d'une démarche participative, de distinguer ce qui est imputable à la démarche elle-même de ce qui est lié au contexte. De fait, la plupart des effets observés sont probablement liés aux deux, puisque si la démarche a suscité une dynamique dans un contexte donné, c'est que cette dynamique appartenait au champ des possibles de ce contexte.

L'un des éléments de méthode les moins neutres fut le choix nominatif des participants invités aux ateliers. Alors que dans de nombreux processus participatifs, les participants viennent sur la base du volontariat, nous avons systématiquement établi une liste d'invités, en négociant cette liste avec des informateurs clefs et les leaders des villages. Il s'agissait de s'assurer que la diversité des intérêts au sein du village serait représentée (les villageois les plus pauvres, peu présents dans la vie politique locale, risquaient sinon d'être insuffisamment représentés), et que tous les acteurs présents à un atelier seraient capables d'y défendre leurs intérêts. La sélection des participants permet aussi d'influencer la création d'éventuelles alliances. Ainsi, à Mae Salaep, le regroupement des agriculteurs sans accès à l'eau, autour du leader religieux, fut déterminant pour permettre à ces derniers de faire valoir leurs intérêts dans les débats. Le choix d'une telle méthode de sélection des participants appelle cependant une réflexion sur la représentativité et la légitimité du groupe convié : si les membres sont désignés par les animateurs du processus pour représenter des catégories d'intérêts données, ils ne constituent pas pour autant de réels représentants, désignés ou élus par un groupe d'intérêts qui se reconnaît en tant que tel.

Le choix des outils fut également loin d'être neutre. En témoigne par exemple le choix du jeu de rôles : accessible à tous, $\mathrm{y}$ compris aux personnes les moins formellement éduquées, le jeu était en outre conçu de façon à mettre en 
évidence la diversité des intérêts en présence, incluant ceux des acteurs les moins influents qui sont les plus susceptibles d'être occultés. À Mae Salaep, par exemple, ce jeu a mis à l'ordre du jour la question de l'inégal accès à l'eau, que les agriculteurs aisés avaient intérêt à passer sous silence. De même, le choix des supports peut aussi favoriser (ou au contraire freiner) l'émergence de processus de négociation intégratifs plutôt que distributifs. À Nan, tant que les supports (plateaux de jeu, interface spatiale du modèle) étaient spatialement explicites, laissant la possibilité de faire apparaître un hypothétique tracé des limites du parc national, les discussions se soldaient systématiquement par une confrontation distributive sur cette frontière-type de négociation de laquelle les acteurs les moins influents ont peu de chance de sortir gagnants. Cen'est que lorsque nous avons proposé aux participants de réfléchir à la gestion d'un espace commun, sans support spatialement explicite, à l'aide de graphes d'évolutions de divers indicateurs, que les discussions purent être plus constructives, sur un mode intégratif, et les intérêts des acteurs les moins influents pris en compte (Barnaud et al., 2013).

Enfin, alterner des entretiens individuels et des discussions en sous-groupes avec les débats en plénière permit à plusieurs reprises aux acteurs les plus faibles de s'exprimer librement, hors de la sphère d'influence de certains participants, et de mieux formaliser leurs arguments.

Tous ces choix méthodologiques ne sont pas neutres. Si cette non-neutralité est ici assumée, il n'en reste pas moins qu'il faut s'interroger sur la légitimité de ces choix aux yeux des acteurs qui en bénéficient ou qui en subissent les conséquences.

\section{Discussion sur la légitimité de la participation dans ses multiples dimensions}

\section{Légitimité sociale d'un processus non neutre vis-à-vis des asymétries de pouvoir}

Dans la posture d'accompagnement critique, la légitimité est considérée comme le fruit d'une coconstruction : celui qui pilote le processus participatif devrait expliciter ses hypothèses et partis-pris, de façon qu'ils puissent être remis en cause ou acceptés comme étant légitimes par les participants. Il s'agit d'une préoccupation non seulement éthique, mais aussi pragmatique car si le processus va à l'encontre des souhaits des acteurs, il a peu de chances d'obtenir des effets durables. Dans notre cas, le principal parti-pris, explicité comme tel, était la volonté de se rapprocher autant que possible d'une forme de concertation équitable, dans laquelle tous les participants auraient une chance de faire entendre et valoir leurs intérêts.
Concrètement, en amont de chaque atelier, les objectifs et les principes méthodologiques du processus étaient discutés avec les acteurs dans le cadre d'entretiens individuels. Il s'agissait de tenir compte de leurs préoccupations en ajustant le processus si nécessaire, mais aussi parfois de les convaincre de leur intérêt potentiel à participer à la démarche proposée. Par exemple, dans le cas de Nan, nous faisions l'hypothèse que le chef du parc national avait intérêt à établir des relations apaisées avec les villageois (dans un contexte où les villageois menaçaient par exemple de déclencher des feux de forêt), et à afficher à Bangkok des pratiques allant dans le «bon » sens, celui de la participation.

Cette posture - expliciter ses partis-pris de façon à interroger et coconstruire la légitimité sociale du processus participatif - a cependant ses limites. Tout d'abord, concrètement, il est illusoire de croire que l'on peut tout expliciter - il y a des partis-pris dont le concepteur n'est pas conscient lui-même - et de penser que les acteurs pourront et oseront tout remettre en cause. C'est donc également par un suivi fin des réactions suscitées par sa démarche que le concepteur peut en évaluer la légitimité. Par ailleurs, on peut se demander si d'un point de vue stratégique, il est pertinent de tout expliciter auprès de tous les acteurs. S'il est nécessaire, dans la posture proposée, de ne pas avoir d'agenda caché (la perte de confiance qui en résulterait serait dommageable), il est souvent opportun de présenter les choses avec diplomatie. Cependant, entre diplomatie et manipulation, les frontières peuvent être minces. Et s'il y a manipulation de certains acteurs, peut-on encore parler de légitimité sociale ? Comment gérer alors le cas d'acteurs refusant de participer au processus? Dans l'expérience de Nan, quelques familles avaient opté pour une stratégie d'intimidation vis-à-vis du parc (menaces verbales et physiques) et n'ont jamais accepté de nous rencontrer ou de se rendre aux ateliers, alors que le reste de la communauté désapprouvait leur stratégie qui renforçait l'hostilité du parc à leur égard et réduisait leur marge de négociation. Certains auteurs critiques suggèrent que, dans de telles situations, il faut mettre en place des mécanismes pour faire pression sur ces acteurs (Leeuwis, 2000). Cette option va cependant à l'encontre de la posture proposée. Faire pression sur des acteurs se justifie dans le cadre d'un processus dont l'objectif est d'aboutir à une prise de décision, quels que soient les moyens nécessaires. Dans notre cas, il s'agit de proposer une arène de concertation reposant sur des principes d'équité et de légitimité, quitte à ce que cette arène ne mène pas à une prise de décision tangible. Nous nous situons dans la lignée d'une posture postnormale qui cherche à améliorer la qualité du processus d'interactions qui mène à la prise de décision, plutôt que la qualité de la décision elle-même (Funtowicz et Ravetz, 1994 ; ComMod, 2005). Cela implique donc de reconnaître que des situations de conflits et de blocage, 
telles que celle qui a été évoquée, constitueraient l'une des limites du cadre d'efficacité de la posture proposée. Cela pose également la question de l'identification des acteurs légitimant le processus : vis-à-vis de qui, de quels acteurs, la démarche doit-elle être légitime ? Vis-à-vis de l'ensemble des acteurs de la société ? De ceux qui sont concernés par ce qui s'y discute? De quelques acteurs clefs qui la soutiennent ? Il y a là autant de réponses possibles que de postures ou de façons de concevoir la légitimité sociale des processus participatifs.

\section{Des pistes de réflexion sur la légitimité scientifique et culturelle de la participation}

Dans cet article, nous avons centré nos propos sur la question de la légitimité sociale des démarches participatives au regard des asymétries de pouvoir. Ces réflexions s'adressent autant à des agents de développement qu'à des chercheurs pilotant des processus participatifs. Cependant, dans le cas des chercheurs, d'autres formes de légitimité peuvent être questionnées.

Un chercheur est en effet mandaté pour produire des connaissances sur un système, pas pour le modifier, comme le serait par exemple un salarié d'une collectivité territoriale en charge d'organiser une concertation sur un territoire. Quelle est alors la légitimité d'un chercheur à intervenir dans ce système? Dans la plupart des démarches de recherche-intervention, cette légitimité s'acquiert en développant des partenariats avec des acteurs clefs qui soutiennent le processus (Mathevet et al., 2010). Dans certains cas, ces acteurs en sont même les commanditaires. Cependant, si un chercheur qui intervient sans être mandaté ou soutenu a une faible légitimité pour intervenir, celui qui est officiellement mandaté par un acteur sera certes fortement légitime aux yeux de cet acteur, mais il pourra en devenir moins légitime aux yeux des autres. Cette réflexion rejoint la question évoquée précédemment : vis-à-vis de qui une démarche participative devrait-elle être légitime?

Le chercheur ne peut notamment pas éluder la question de sa légitimité à l'égard de la communauté scientifique. Si la recherche fondamentale considère parfois la recherche appliquée comme non légitime car instrumentalisée, que dire alors de la recherche-intervention? Comme le soulignent Casablanca et Albaladejo (1997), on peut se demander « en quoi le fait de se mettre au service d'un ou plusieurs acteurs du développement ou même devenir acteur soi-même permet [...] de faire autre chose que du développement intelligent». Quelles sont les connaissances produites dans l'action ? Quelle est leur validité hors du champ de l'action ? La généralisation de ces connaissances par essence situées est en effet souvent un exercice difficile (Soulard et al., 2007).

Rappelons par ailleurs que la participation repose sur des théories du changement social développées dans des pays occidentaux, en Europe (Habermas, 1987), aux États-Unis (Lewin, 1951), ou encore en Amérique latine (Freire, 1983). On peut alors interroger la légitimité culturelle des démarches participatives conduites hors du berceau des idéologies qui en sous-tendent les principes. Cela ne revient-il pas paradoxalement à imposer la participation ? Plusieurs éléments permettent de lever ce paradoxe, au moins en partie. Tout d'abord, dans le cas de la Thaïlande, l'idéal démocratique a été importé depuis plusieurs décennies et fait désormais partie du courant de pensée dominant (Arghiros, 2001). En outre, la posture proposée dans cet article est justement fondée sur la volonté de ne rien imposer aux acteurs. Si les acteurs n'avaient pas adhéré à la démarche, s'ils n'avaient pas exprimé un intérêt à y participer, ellen'aurait pas débuté ou aurait avorté. Cependant, si cette réponse permet d'assurer au lecteur qu'il n'y a pas eu ingérence, elle n'est pas tout à fait satisfaisante sur le plan intellectuel, car elle repose dans le fond sur l'idée que la participation est vertueuse. Cooke et Kothary (2001) suggèrent ainsi qu'une approche réellement réflexive sur la participation ne peut être menée que par des individus qui ne partagent pas ce postulat. D'une façon plus générale, ce débat renvoie à certains dilemmes en éthique du développement, liés au fait que toute action de développement a des effets positifs et négatifs. Kahn et al. (2007) proposent pour y faire face une " éthique de la discussion ", qui entre fortement en résonnance avec l'idée d'une légitimité coconstruite développée dans cet article.

\section{Conclusion}

Au final, interroger la légitimité des démarches participatives s'avère être un exercice à la fois nécessaire et délicat. La prise en compte des asymétries de pouvoir dans un processus participatif relève ainsi d'un travail d'équilibriste dans une situation permanente d'équilibre instable. Celui-ci est tiraillé entre, d'un côté, une volonté de laisser le collectif d'acteurs guider le processus en ne faisant que l'accompagner - au risque que le processus soit manipulé par les acteurs les plus influents - et, de l'autre, le souci de structurer l'espace d'échanges pour éviter que la voix des acteurs les moins influents ne soit occultée - au risque d'affaiblir la légitimité sociale du processus qu'il met en œuvre. Avançant sur cette ligne de crête, le concepteur d'un processus participatif doit à chaque pas interroger et réinventer les conditions de son équilibre. Qu'il s'agisse d'interroger la légitimité sociale, culturelle ou scientifique des démarches participatives, les pistes de réflexion proposées dans cet article n'apportent donc pas de solution clefs en main. Elles constituent au mieux un balancier pour l'équilibriste : des éléments de réflexion permettant d'alimenter la continuelle remise en question nécessaire lors de la mise en œuvre d'une démarche participative. 


\section{Remerciements}

Ces travaux ont été réalisés dans le cadre d'une thèse financée par une allocation du ministère de l'Éducation nationale, de l'Enseignement supérieur et de la Recherche (MENESR), avec le soutien de la direction scientifique du Cirad, et de la participation à des programmes de recherche ANR ADD-ComMod (financement ANR) et CPWF PN 25. L'auteur tient par ailleurs à remercier Anne-Marie Van Paassen, François Bousquet et Guy Trébuil pour leur contribution aux réflexions menées dans le cadre de cette thèse; Nicolas Becu et Olivier Barreteau pour leurs suggestions à la lecture de cet article; ainsi que deux relecteurs anonymes pour leur relecture critique et constructive.

\section{Références}

Arghiros, D., 2001. Democracy, Development and Decentralization in Provincial Thailand, Richmond (Surrey), Curzon \& Nordic Institute of Asian Studies.

Argyris, C., 1995. Savoir pour agir: surmonter les obstacles à l'apprentissage organisationnel, Paris, InterÉditions.

Avenier, M.-J., Schmitt, C. (Eds), 2007. La Construction de savoirs pour l'action, Savoir \& Action, Rencontres, Paris, L'Harmattan.

Barbier, R., 2005. Quand le public prend ses distances avec la participation: topiques de l'ironie ordinaire, Natures Sciences Sociétés, 13, 258-265.

Barnaud, C., D'Aquino, P., Daré, W., Fourage, C., Mathevet, R., Trébuil, G., 2010a. Les asymétries de pouvoir dans les processus d'accompagnement, in Étienne, M. (Ed.), La Modélisation d'accompagnement : une démarche participative en appui au développement durable, Paris, Quae, 125-152.

Barnaud, C., Page, C. L., Dumrongrojwatthana, P., Trébuil, G., 2013. Spatial representations are not neutral: Lessons from a participatory agent-based modelling process in a land-use conflict, Environmental Modelling \& Software, 45, 150-159.

Barnaud, C., Promburom, T., Trébuil, G., Bousquet, F., 2007. An evolving simulation and gaming process to facilitate adaptive watershed management in mountainous Northern Thailand, Simulation and Gaming, 38, 398-420.

Barnaud, C., Trébuil, G., Dumrongrojwatthana, P., Marie, J., 2008a. Area study prior to companion modelling to integrate multiple interests in upper watershed management of Northern Thailand, Southeast Asian Studies, 45, 4, 559-585.

Barnaud, C., Trébuil, G., Promburom, P., Bousquet, F., $2008 \mathrm{~b}$. La modélisation d'accompagnement pour une gestion concertée des ressources renouvelables en Thaillande, Économie Rurale, 303/304/305, 39-59.

Barnaud, C., Van Paassen, A., Trébuil, G., Promburom, T., Bousquet, F., 2010b. Dealing with power games in a companion modelling process: Lessons from community water management in Thailand highlands, Journal of International Agricultural and Extension Education, 16, 1, 55-74.

Barreteau, O., Bousquet, F., Attonaty, J., 2001. Role-playing games for opening the black box of multi-agent systems: Method and lessons of its application to Senegal River valley irrigated systems, Journal of Artificial Societies and Social Simulation, 4, 2 (online: http:/ /jasss.soc.surrey.ac.uk/4/2/5.html).

Becu, N., Neef, A., Schreinemachers, P., Sangkapitux, C., 2008. Participatory modeling to support collective decisionmaking: Potential and limits of stakeholder involvement, Journal of Land Use Policy, 25, 498-509, doi:10.1016/ j.landusepol.2007.11.002.

Boltanski, L., Thévenot, L., 1991. De la justification : les économies de la grandeur, Paris, Gallimard.

Borrini-Feyerabend, G., Pimbert, M., Farvar, M.T., Kothari, A., Renard, Y., 2004. Sharing Power: Learning by Dowing in Comanagement of Natural Resources Throughout the World, Cenesta, Tehran, IIED, IUCN/CEESP/CMWG.

Boulding, K.E., 1989. Three Faces of Power, Newbury Park (CA), Sage Publications.

Bousquet, F., Barreteau, O., d'Aquino, P., Etienne, M., Boissau, S., Aubert, S., Le Page, C., Babin, D., Castella, J.C., 2002. Multi-agent systems and role games: Collective learning processes for ecosystem management, in Janssen, M. (Ed.), Complexity and Ecosystem Management: The Theory and Practice of Multi-agent Approaches, Cheltenham, Edward Elgar Publishers, 248-284.

Bousquet, F., Barreteau, O., Mullon, C., Weber, J., 1996. Modélisation d'accompagnement : systèmes multi-agents et gestion des ressources renouvelables. Communication au colloque Quel environnement au XXI siècle? Environnement, maîtrise du long terme et démocratie, Abbaye de Frontevraud, 8-11 septembre.

Carnevale, J.P., 2006. Creativity in the outcomes of conflict, in Deutsch, M., Coleman, P.T., Marcus, E.C, Handbook of Conflict Resolution: Theory and Practice, 2nd edition, San Francisco, Jossey-Bass, 414-435.

Casabianca, F., Albaladejo, C., 1997. Des multiples légitimités de la recherche-action, in Albaladejo, C., Casabianca, F., La Recherche-action : ambitions, pratiques, débats,Versailles, Inra éditions.

Chambers, R., Pacey, A., Thrupp, L.A., 1989. Farmer First: Farmer Innovation and Agricultural Research, London, Intermediate Technology Publications.

Chanal, V., Lesca, H., Martinet, A.-C., 1997. Recherche ingénierique et connaissances procédurales en sciences de gestion : réflexions épistémologiques et méthodologiques, Revue Française de Gestion, 116, 41-51.

Checkland, P., 1981. Systems Thinking, Systems Practice, Chichester, John Wiley and Sons.

ComMod (collectif), 2005. La modélisation comme outil d'accompagnement, Natures Sciences Sociétés, 13, 165-168.

Cooke, B., Kothari, U. (Eds), 2001. Participation: The New Tyranny?, London, New York, Zed Books.

D'Aquino, P., 2009. La participation comme élément d'une stratégie globale d'intervention: l'approche "gestion autonome progressive », Cahiers Agricultures, 18, 5, 433-440.

Daré, W., Aubert, S., Bah, A., Botta, A., Diop Gaye, I., Fourage, C., Lajoie, G., Leclerc, G., 2008. Difficultés de la participation en recherche-action : retour d'expériences de modélisation d'accompagnement en appui à l'aménagement du territoire, VertigO, 8, 1, 22.

Daré, W., Fourage, C., Gaye, I.D., 2007. Positionnement des sociologues dans la démarche de modélisation Domino, Nouvelles Perspectives en Sciences Sociales, 2, 2, 103-127. 
Edmunds, D., Wollenberg, E., 2001. A strategic approach to multistakeholder negociations, Development and Change, 32, 231-253.

Eversol, R., 2003. Managing the pitfalls of participatory development: Some insight from Australia, World Development, 31, 5, 781-795.

Faysse, N., 2006. Troubles on the way: An analysis of the challenges faced by multistakeholder platforms, Natural Resources Forum, 30, 219-229.

Freire, P., 1983. Pédagogie des opprimés, suivi de Conscientisation et révolution, Paris, Maspero.

Funtowicz, S.O., Ravetz, J.R., 1994. The worth of a songbird: Ecological economics as a post-normal science, Ecological Economics, 10, 3, 197-207.

Giddens, A., 1984. The Constitution of Society: Outline of the Theory of Structuration, Cambridge (UK), Polity Press.

Guijt, I., Shah, M., 1998. Waking up to power, conflict and process, in Guijt, I., Shah, M., The Myth of Community: Gender Issues in Participatory Development, London, Intermediate Technology Publications.

Habermas, J., 1987. Théorie de l'agir communicationnel, Paris, Fayard.

Innes, J.E., 2004. Consensus building: Clarifications for the critics, Planning Theory, 3, 1, 5-20.

Jackson, M.C., 2000. Systems Approaches to Management, New York, Kluwer Academic, Plenum Publishers.

Johnson, N., Lilja, N., Ashby, J. A., Garcia, J. A., 2004. The practice of participatory research and gender analysis in natural resource management, Natural Resources Forum, 28, 3, 189-200.

Kahn, F., Lecourt, D., Moulin, A.-M. (Eds), 2007. Y a-t-il une éthique propre à la recherche pour le développement?, Paris, IRD.

Lavigne-Delville, P., Selamna, N. E., Mathieu, M. (Eds), 2000. Les Enquêtes participatives en débat: ambition, pratiques et enjeux, Paris, Karthala, Icra, Gret.

Leeuwis, C., 2000. Reconceptualizing participation for sustainable rural development: Towards a negotiation approach, Development and Change, 31, 931-959.

Lewin, K., 1951. Field Theory in Social Science: Selected Theoretical Papers, New York, Harper and Row.

Liu, M., 1997. Fondements et pratiques de la recherche-action, Paris, L'Harmattan.

Mathevet, R., Antona, M., Barnaud, C., Fourage, C., Trébuil, G., Aubert, S., 2010. Contextes et dépendances des contextes d'accompagnement, in Etienne, M., La Modélisation d'accompagnement: une démarche participative en appui au développement durable, Paris, Quae, 103-123.

Mazoyer, M., Roudart, L., 1997. Histoire des agricultures du monde : du néolithique à la crise contemporaine, Paris, Seuil.

Mermet, L., Billé, R., Leroy, M., Narcy, J.-B., Poux, X., 2005. L'analyse stratégique de la gestion environnementale : un cadre théorique pour penser l'efficacité en matière d'environnement, Natures Sciences Sociétés, 13, 127-137.
Mormont, M., 2009. Le sociologue dans l'action collective face au risque, Développement Durable et Territoires (online: http:/ /hdl.handle.net/2268/25238).

Munoz, I., Paredes, M., Thorp, R., 2007. Group inequalities and the nature and power of collective action: Case studies from Peru, World Development, 35, 11, 1929-1946.

Neef, A. (Ed.), 2005. Participatory Approaches for Sustainable Land Use in Southeast Asia, Bangkok, White Lotus.

Nelson, N., Wright, S., 1995. Participation and power, in Nelson, N., Wright, S., Power and Participatory Development: Theory and Practice, London, Intermediate Technology Publications, 1-18.

Ostrom, E., Gardner, R., Walker, J., 1994. Rules, games $\mathcal{E}$ common-pool resources, Michigan, University of Michigan Press.

Petit, S., Fleury, P., Michel, V., Mougenot, C., 2008. Raconter la recherche-intervention: retour sur trois opérations de gestion de la biodiversité, Natures Sciences Sociétés, 16, 326336.

Pretty, J., 1998. Participatory learning for integrated farming, in Foo, E.-L., Senta, T.D. (Eds), Integrated Bio-Systems in Zero Emissions Applications: Proceedings of the Internet Conference on Integrated Bio-Systems.

Rawls, J., 1997. Théorie de la justice, Paris, Seuil.

Ribot, J.C., 2001. Integral local development: "accomodating multiple interests" through entrustment and accountable representation, International Journal of Agricultural Resources, Governance and Ecology, 1, 3/4, 327-350.

Röling, N., Wagemakers, M. A. (Eds), 1998. Facilitating Sustainable Agriculture: Participatory Learning and Adaptive Management in Times of Environmental Uncertainty, Cambridge (UK), Cambridge University Press.

Sato, J., 2003. Public land for the people: The institutional basis of community forestry in Thailand. Journal of Southeast Asian Studies, 34, 2, 329-346.

Soulard, C.-T., Compagnone, C., Lémery, B., 2007. La recherche en partenariat: entre fiction et friction, Natures Sciences Sociétés, 15, 1, 13-22.

Stassart, P.M., Mormont, M., Jamar, D., 2008. La rechercheintervention pour une transition vers le développement durable, Économie Rurale, 306, 8-22.

Trébuil, G., Shinawatra-Ekasingh, B., Bousquet, F., ThongNgam, C., 2002. Multi-agent systems companion modelling for integrated watershed management: A Northern Thailand experience, in Jianchu, X., Mikesell, S., Landscapes of Diversity:Proceedings of 3rd International Conference on Montane Mainland Southeast Asia (MMSEA 3), Lijiang, Yunnan, Yunnan Science and Technology Press, 25-28 August, 349-358.

Ulrich, W., 1983. Critical Heuristics of Social Planning: A New Approach to Practical Philosophy, Bern, Haupt.

Wollenberg, E., Anderson, J., Edmunds, D., 2001. Pluralism and the less powerful: Accommodating multiple interests in local forest management, International Journal of Agricultural Resources, Governance and Ecology, 1, 3/4, 199-222.

Reçu le 10 novembre 2011. Accepté le 5 juillet 2012. 